\title{
Study of the Electrocatalytic Reaction of Hydrogen Peroxide Mediated by Azobenzene Monolayer with Several Multipotential Pulse Techniques
}

\author{
J. González, C. M. Soto, A. Molina* \\ Departamento de Quimica Física, Universidad de Murcia, Espinardo 30100, Murcia, Spain
}

\begin{abstract}
The comparison between the response corresponding to a simple charge transfer taking place at an electroactive monolayer with that of an electrocatalytic process at a modified electrode are presented by using the multipulse potential techniques cyclic staircase voltammetry (CSCV) and cyclic square wave voltammetry (CSWV). General analytical and explicit equations for the $I-E$ responses have been tested by means of the study of the reduction of azobenzene monolayer at mercury electrode. The value of the chemical rate constant $\left(k_{c}^{\prime}=40 M^{-1} s^{-1}\right)$ has been easily obtained from the cathodic plateau of the CSCV curves and from the best fitting between theoretical and experimental CSWV curves.
\end{abstract}

Keywords: Azobenzene, hydrogen peroxide, catalytic mechanism, monolayer, multipotential pulse techniques.

\section{INTRODUCTION}

The development of monolayer-functionalized surfaces has been a successful trend in the characterization and control of electrochemical reaction kinetics and catalysis [14]. The modification of the electrode surface can be carried out by different routes, the covalent attachment of redox species being one of the most used [1,2, 5-7].

Azobenzene-terminated self-assemble monolayers are extensively used in studies of electron-transfer processes [8]. The chemical composition, structure and length of the molecules linked to the surface via mercury electrode as well as the dielectric environment on the surface are all crucial factors determining the dynamics of the electron transfer between the azobenzene and the electrode surface [9].

Among the broad variety of electrochemical techniques, cyclic multipotential pulse techniques, such as CSCV and CSWV, are powerful tools for the characterization of the response of species immobilized on the electrode surface [2, 10-15]. Those techniques are based on the application of a sequence of potential pulses of discrete nature and, especially, CSWV, present several advantages, one of the most important being their widely acknowledged inherent ability to decouple faradic currents from non faradaic ones [15-17].

Analytical and easy manageable expressions for the current of a simple charge transfer process and of an electrocatalytic process at mediator modified electrodes for any multipotential pulse technique were deduced in previous papers $[13,14,18,19]$.

In order to check the validity of the above mentioned expressions and characterize the azobenzene/ hydrogen peroxide system, we have analysed and compared the response corresponding to the reduction of the azobenzene at

*Address correspondence to this author at the Departamento de Química Física, Universidad de Murcia, Espinardo 30100, Murcia, Spain; Tel: 0034 968 367524; Fax: 0034968 364148; E-mail: amolina@um.es a mercury electroactive monolayer with that of the redox reaction of azobenzene in the presence of hydrogen peroxide as oxidizing agent. From our results, we have observed that azobenzene presents strong adsorption, behaves as quasireversible at a mercury electrode and is also influenced by $\mathrm{pH}$ conditions.

\section{EXPERIMENTAL SECTION}

\subsection{Materials and Procedures}

Ethanol, $\mathrm{KNO}_{3}, \mathrm{H}_{2} \mathrm{O}_{2}$ (Merck, reagent grade), and sodium citrate tribasic dehydrate $\left(\mathrm{Na}_{3} \mathrm{C}_{6} \mathrm{H}_{5} \mathrm{O}_{7} \cdot 2 \mathrm{H}_{2} \mathrm{O}\right)$, citric acid $\left(\mathrm{C}_{6} \mathrm{H}_{8} \mathrm{O}_{7}\right)$ and azobenzene (AZB) (Aldrich, reagent grade) were used as received. AZB was dissolved in ethanol, and then diluted with water until the final proportion of ethanol was $1 \%$. AZB was adsorbed at the mercury surface at a rest potential $E_{\text {rest }}=-0.200 \mathrm{~V}$ versus reference for $10 \mathrm{~s}$ prior to measurements such that all the measurements were taken when the surface coverage of this species had reached equilibrium with the bulk concentration.

The low azobenzene concentration used $(30 \mu M)$ guarantees that any possible contribution to the whole response due to dissolved azobenzene can be considered as negligible. Moreover, we have checked that desorption has not taken place in the time scale of the experiments (see also $[20,21])$.

\subsection{Aparatus}

CSCV and CSWV were performed by using a computerdriven potentiostat-galvanostat designed and constructed by Quiceltron.

A three-electrode cell was employed in the experiments. A homemade static mercury drop electrode (SMDE) served as working electrode. The electrode radius of the SMDE was determined by weighing a large number of drops. The counter electrode was a Pt foil and the reference electrode was a $\mathrm{Ag} / \mathrm{AgCl}, \mathrm{KCl}$ (saturated) electrode. Solutions were prepared with distilled deionized water (Milli-Q filtering system). Nitrogen gas was passed through solutions for deaeration for $20 \mathrm{~min}$ prior to measurements, with nitrogen 
atmosphere maintained over the solution during all the experiments. All the kinetic and thermodynamic values obtained for the AZB system correspond to series of five essays. The results are the mean of the five experimental values. The errors correspond to the standard deviation.

To avoid transport effects in the catalytic response, a magnetic stirrer with a constant rotation rate was placed close to the electrode surface, so a constant agitation was maintained during the whole experiment. By proceeding in this way we have checked that the values of the catalytic pseudo first order rate constant obtained do not depend on the $\mathrm{H}_{2} \mathrm{O}_{2}$ concentration.

\section{RESULTS AND DISCUSSION}

\subsection{E mechanism at a Modified Electrode}

In previous papers we have deduced an analytical and easy manageable expression for the current corresponding to a simple charge transfer process of any reversibility degree and any sequence of consecutive potential steps designed by $E_{1}, E_{2}, \ldots, E_{p-1}, E_{p}$, of the same length $\tau$. This expression is applied to the following process, $A+n e^{-} \underset{k_{o x}}{\stackrel{k_{r e d}}{\rightleftarrows}} B$, where the redox couple $\mathrm{A} / \mathrm{B}$ is immobilized at the electrode surface $[13,14,22,23]$ and $k_{r e d}$ and $k_{o x}$ are the electrochemical rate constants of the forward (reduction) and backward (oxidation) heterogeneous processes, respectively $[14,15]$ :

$\frac{I_{p}}{Q_{\text {MAX }}}=k_{r e d, p}-k_{E, p} \sum_{j=1}^{p-1}\left(\frac{k_{r e d, j}}{k_{E, j}}\left(1-\theta_{j}\right) \prod_{l=j+1}^{p-1} \theta_{l}\right) \theta_{p}$

with

$\theta_{p}=\exp \left(-k_{E, p} \tau\right)$

$k_{E, p}=k_{r e d, p}+k_{o x, p}$

$Q_{M A X}=n F S \Gamma_{T}$, where $S$ is the electrode area, $n$ the number of transferred electrons and $\Gamma_{T}$ the total electroactive surface excess.

Eq. (1) is applicable for different multipotential step techniques. In this paper we will apply this equation to CSCV and CSWV which are among those most frecuently used experimentally.

The potential sequence corresponding to SCV technique is a staircase potential of the form $[2,24]$ :

$E_{m}=E_{\text {initial }}+(m-1) \Delta E ; 1 \leq m \leq p$

with $p$ being the total number of potential steps and $\Delta E$ the pulse amplitude, whereas the potential sequence corresponding to SWV can be described by the following equation:

$\left.\begin{array}{rlr}E_{p}=E_{\text {intital }}+\left[\operatorname{Int}\left(\frac{p+1}{2}\right)-1\right]\left|\Delta E_{s}\right|+(-1)^{p+1}\left|E_{S W}\right| ; p=1,2, \ldots, n p / 2 \\ E_{p}=E_{n p-p+1} ; & p=(n p / 2)+1, \ldots, n p\end{array}\right\}$ where $\left|\Delta E_{s}\right|$ is the potential step in the staircase and $\left|E_{S W}\right|$ is the square wave amplitude, in agreement with the scheme given in references $[15,16,25,26]$. The waveform can be applied in single (direct scan only) or cyclic mode (direct and inverse scans). In the latter, once the final potential has been reached, the waveform is reversed, returning through the same sequence of pulses to the initial potential.

The signal is measured at the end of each potential pulse and the net response is the difference between the signal corresponding to a pulse with odd index (forward) and the signal of the following pulse with even index (reverse). Typically, this signal is the current, such that the usual $I_{S W}-E$ square wave voltammetry curve is defined as:

$I_{S W}=I_{2 p-1}-I_{2 p}=I_{f}-I_{r} ; p=1,2, \ldots,(n p / 2)$

The subindexes $f$ and $r$ in the above expression refer to the forward and reverse pulses (equal to $2 p-1$ and $2 p$, respectively).

$I_{S W}$ signal is plotted versus the index potential, defined as an intermediate potential value between each pair of consecutive pulses,

$$
E_{\text {index }, p}=E_{p}+(-1)^{p}\left|E_{S W}\right|
$$

In order to corroborate these Equations, we have analyzed the experimental behaviour of azobenzene monolayers in aqueous medium on a mercury electrode surface. Azobenzene/hydrazobenzene is a well-known redox couple where both components are strongly adsorbed on a mercury electrode surface. The redox transformation in this medium involves an electrochemically quasi-reversible exchange of two electrons and two protons. This redox reaction is widely used for illustrations of different theoretical models, such as surface redox processes, and electrode reactions with coupled adsorption phenomena in general $[20,21,27,28]$.

Fig. (1) shows the experimental (white circles) and theoretical (solid lines) $I_{S W}-E$ curves corresponding to the system azobenzene $30 \mu \mathrm{M}+0.5 \mathrm{M} \mathrm{KNO}_{3}(\mathrm{pH}=4.28)$ (simple charge transfer process) obtained in CSWV, for $\tau=10 \mathrm{~ms}$ and for different values of $E_{S W}$.

The $I_{S W}-E$ curves of Fig. (1) show that the cathodic and anodic peaks are almost symmetrical and practically centred on the formal potential (dotted line). Note that the $I_{S W}-E$ curves present a single peak for low values of $E_{S W}$, that becomes into two peaks as $E_{S W}$ increases. Moreover, the separation between them increases with $E_{S W}$. The characteristic of peak features observed in this Figure informs us that the electrochemical transfer process is quasireversible [16].

We have also included different theoretical curves (solid lines) calculated by using Eqs. (1) and (6). Once we 
determined the formal potential $E^{0}=(-0.123 \pm 0.002) \mathrm{V} v s$ SCE. (dotted line), the best fittings were obtained by using the following parameters: $k^{0}=(19 \pm 2) \mathrm{s}^{-1}, n=2, \alpha=0.5$ and $Q_{\text {MAX }}=0.110 \pm 0.03 \mu \mathrm{C}$.

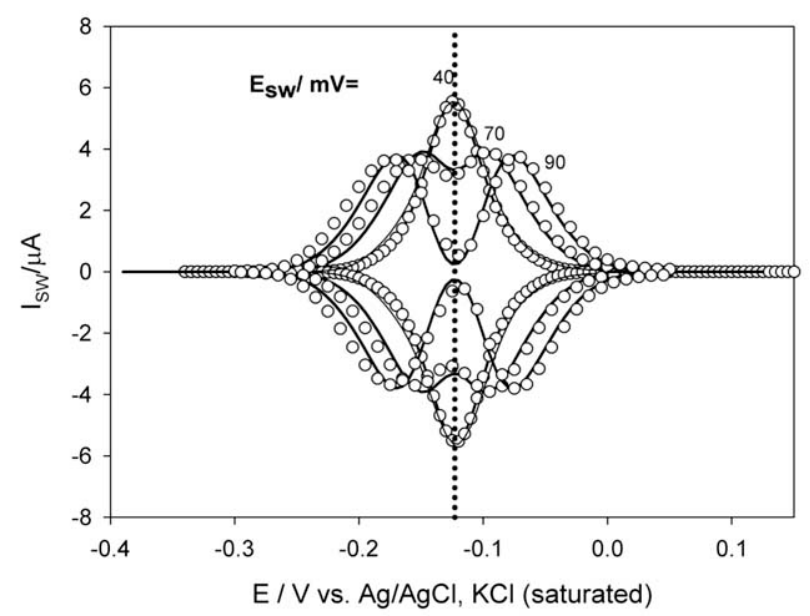

Fig. (1). (white circles) Experimental $I_{S W}-E$ curves corresponding to the system azobenzene $30 \mu M+0.5 \mathrm{M} \mathrm{KNO}_{3}$ $\mathrm{pH}=4.28$ (E mechanism) on a mercury electrode of radius $r_{0}=0.02 \mathrm{~cm} . \tau=10 \mathrm{~ms}$. The values of the $E_{S W}$ are on the curves (in $\mathrm{mV}$ ). (solid lines) Theoretical $I_{S W}-E$ curves calculated for a simple charge transfer process (Eqs. (1) and (6)) by using the following parameters: $E^{0}=-0.123 \mathrm{~V}, k^{0}=19 \mathrm{~s}^{-1}, n=2, \alpha=0.5$, $Q_{\text {MAX }}=0.110 \mu \mathrm{C}$ and $\mathrm{T}=298 \mathrm{~K}$. Dotted line marks the formal potential position.

\subsection{Catalytic Mechanism at a Modified Electrode}

We will now consider the study of the electrochemical response of an electrocatalytic process taking place at a modified redox mediator in agreement with the Scheme:

$$
A+n e^{-} \underset{k_{o x}}{\stackrel{k_{r e d}}{\rightleftarrows}} B+C \stackrel{k_{c}^{\prime}}{\longrightarrow} A+D
$$

where $A$ and $B$ refer to strongly adsorbed species and $C$ and $D$ to species in solution. The catalytic rate constant is $k_{c}=k_{c}^{\prime} c_{C}^{*}$ where $k_{c}^{\prime}$ is the true chemical rate constant and $c_{C}^{*}$ is the surface concentration of $\mathrm{C}$ species, which is considered as constant and equal to the bulk concentration.

The expression of the current-potential curves in staircase voltammetry, for any reversibility degree of the charge transfer of the electrocatalytic process, is given by [19]:

$$
\begin{aligned}
\frac{I_{p}}{Q_{M A X}}= & -\left(k_{T, p}-k_{c}\right) \sum_{j=1}^{p-1}\left(\frac{k_{r e d, j}}{k_{T, j}}\left(1-\theta_{j}\right) \prod_{l=j+1}^{p} \theta_{l}\right) \\
& +\frac{k_{r e d, p}}{k_{T, p}}\left(k_{T, p}-k_{c}\right) \theta_{p}+k_{c} \frac{k_{r e d, p}}{k_{T, p}}
\end{aligned}
$$

where
$k_{T, p}=k_{E, p}+k_{c}$

and $\vartheta_{p}=\exp \left(-k_{T, p} \tau\right)$

Note that we obtain the current-potential curves in square wave voltammetry by introducing Eq. (8) in (6).

In this paper, the redox reaction of azobenzene was also investigated in the presence of hydrogen peroxide as oxidizing agent. Hydrazobenzene, formed by electrochemical reduction of azobenzene, is reoxidized by hydrogen peroxide, and the whole electrode reaction turns into a surface catalytic mechanism in according to the next scheme $[19,27]$ :

$$
\left.\begin{array}{l}
P h-N=N-P h+2 e^{-}+2 H^{+} \underset{k_{o x}}{\stackrel{k_{c d}}{\rightleftarrows}} P h-N H-N H-P h \\
P h-N H-N H-P h+H_{2} \mathrm{O}_{2} \stackrel{k_{c}^{\prime}}{\longrightarrow} P h-N=N-P h+2 H_{2} O
\end{array}\right\}
$$

In Fig. (2) we have plotted the experimental (circles) and theoretical (solid lines) $I_{S W}-E$ curves corresponding to the system azobenzene $30 \mu \mathrm{M}+0.5 \mathrm{M} \mathrm{KNO}_{3}+0.1 \mathrm{M}$ Citric acid/sodium citrate without $\mathrm{H}_{2} \mathrm{O}_{2}$ (white circles) (E mechanism) and with $25 \mathrm{mM} \mathrm{H}_{2} \mathrm{O}_{2}$ (black circles) (catalytic mechanism) $(\mathrm{pH}=3.22)$. All these curves have been obtained for the same time $\tau=1.0 \mathrm{~ms}$. In the CSWV curves we have used different square wave pulse amplitudes $E_{S W}$ (in $\mathrm{mV}$ ) which are indicated in the figure.

The $I_{S W}-E$ curves for the catalytic mechanism in Fig. (2, black circles) present a single peak for low values of $E_{S W}$, which split in two as $E_{S W}$ increases, and also the separation between them is higher with $E_{S W}$. The peak heights of these curves are almost double those obtained for the E process (white circles) (see Fig. 1).

The formal potential (dotted line) for a catalytic process does not differ from that corresponding to the simple charge transfer reaction. Note that, the different value of the formal potential of Figs. $(\mathbf{1}, \mathbf{2})$ is due to the different conditions of them. The lower $\mathrm{pH}$ value in Fig. (2) gives rise to a more anodic value of formal potential, and also to a higher reversibility as we will see below.

We have also included different theoretical curves (solid lines) calculated from Eqs. (1), (6) and (8). Once the formal potential $E^{0}=(-0.0715 \pm 0.0015) \mathrm{V}$ vs SCE. (dotted line) was determined, the best fittings were obtained with the following parameters: $k^{0}=480 \mathrm{~s}^{-1}, n=2, \alpha=0.5$ and $Q_{\text {MAX }}=0.0159 \pm 0.001 \mu \mathrm{C}$ being $k_{c}=1 \mathrm{~s}^{-1}$ for the catalytic curves.

In Fig. (3) we have studied the effect of the sweep rate $v$ on the experimental $I-E$ curves in $\mathrm{CSCV}$ corresponding to the system azobenzene $30 \mu \mathrm{M}+0.5 \mathrm{M} \mathrm{KNO}_{3}+0.1 \mathrm{M}$ Citric acid/sodium citrate without $\mathrm{H}_{2} \mathrm{O}_{2}$ (white circles) (E mechanism) and with $100 \mathrm{mM} \mathrm{H}_{2} \mathrm{O}_{2}$ (black circles) (catalytic mechanism $)(\mathrm{pH}=3.22)$, respectively, in $\mathrm{CSCV}$ with $\Delta \mathrm{E}=5 \mathrm{mV}$. 


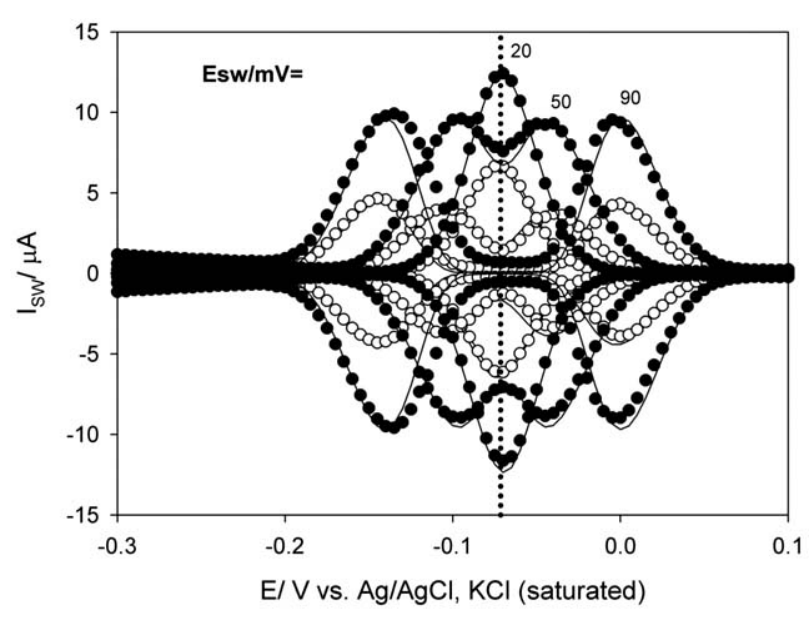

Fig. (2). Experimental $I_{S W}-E$ curves corresponding to the system azobenzene $30 \mu M+0.5 \mathrm{M} \mathrm{KNO}_{3}+0.1 \mathrm{M}$ Citric acid/sodium citrate without $\mathrm{H}_{2} \mathrm{O}_{2}$ (simple charge transfer process) (white circles) and with $25 \mathrm{mM} \mathrm{H}_{2} \mathrm{O}_{2}$ (black circles) (electrocatalytic process) $(\mathrm{pH}=3.22)$ adsorpted on a mercury electrode of radius $r_{0}=0.02 \mathrm{~cm} . \tau=1.0 \mathrm{~ms}$. The values of the square wave pulse amplitude $E_{S W}$ (in $\mathrm{mV}$ ) are on the curves. (solid lines) Theoretical $I_{S W}-E$ curves (Eqs.(1), (6) and (8)) calculated by using the following parameters: $k^{0}=480 s^{-1}, E^{0}=-0.0715 \mathrm{~V}, \quad n=2$, $Q_{\text {MAX }}=0.0159 \mu \mathrm{C}$ and $T=298 \mathrm{~K}$ being $k_{c}=1 \mathrm{~s}^{-1}$ for the catalytic curves. Dotted line marks the formal potential position.

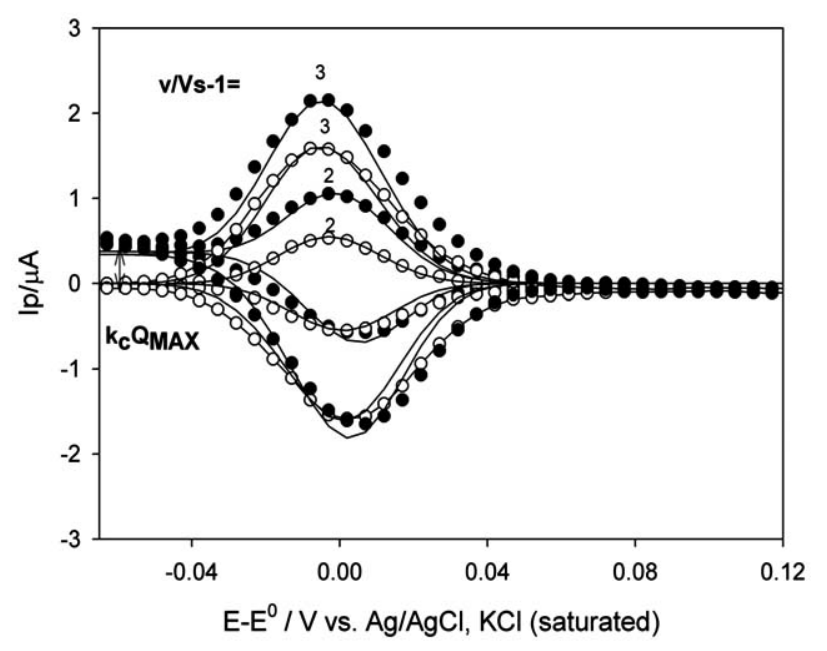

Fig. (3). Experimental $I-E$ curves in CSCV corresponding to the system azobenzene $30 \mu \mathrm{M}+0.5 \mathrm{M} \mathrm{KNO}_{3}+0.1 \mathrm{M}$ Citric acid/sodium citrate without $\mathrm{H}_{2} \mathrm{O}_{2}$ (simple charge transfer process) (white circles) and with $100 \mathrm{mM} \quad \mathrm{H}_{2} \mathrm{O}_{2}$ (black circles) (electrocatalytic process) $(\mathrm{pH}=3.22)$ adsorpted on a mercury electrode of radius $r_{0}=0.02 \mathrm{~cm} . \Delta E=5 \mathrm{mV}$. The values of sweep rate $v$ (in $\mathrm{Vs}^{-1}$ ) are on the curves. (solid lines) Theoretical $I-E$ curves (Eqs. (1) and (8)) calculated by using the following parameters: $k^{0}=480 \mathrm{~s}^{-1}, n=2, Q_{\text {MAX }}=0.078 \mu \mathrm{C}$ and $T=298 \mathrm{~K}$ being $k_{c}=4 s^{-1}$ for the catalytic curves.
From the curves in Fig. (3) it can be seen that in both mechanisms the decrease of the sweep rate $v$ leads to a decrease of the current. Nevertheless, for the catalytic mechanism the peak heights are higher than those of an $\mathrm{E}$ mechanism and also the current reaches the value $k_{c} Q_{M A X}$ at cathodic potentials, independently of the sweep rate [19, 29].

To check the goodness of the data obtained from the experimental $I-E$ curves we have included in Fig. (3) the theoretical ones obtained in CSCV, calculated from Eqs. (1) and (8), which correspond to a simple charge transfer process and a catalytic process, respectively (solid lines). By taking into account the charge transfer constant obtained in the same conditions $\left(k^{0}=480 \mathrm{~s}^{-1}\right)$, the theoretical curves have been calculated using the following parameters: $n=2$, $\alpha=0.5, Q_{\text {MAX }}=0.078 \mu \mathrm{C}$ and being $k_{c}=4 \mathrm{~s}^{-1}$ for the catalytic mechanism. The agreement between theoretical and experimental results confirms the accuracy of the experimental parameters deduced. From the value of $k_{c}$ used, we can finally obtain that the true chemical rate constant of the mechanism is $k_{c}^{\prime}=40 \mathrm{M}^{-1} \mathrm{~s}^{-1}$ [19].

\section{CONCLUSIONS}

The experimental study of Azobenzene $+\mathrm{H}_{2} \mathrm{O}_{2}$ system with the multipulse potential techniques CSCV and CSWV has led to check analytical and explicit expressions previously deduced by us and to obtain electrochemical and chemical information of this system in a very simple way.

\section{ACKNOWLEDGEMENTS}

The authors greatly appreciate the financial support provided by the Dirección General de Investigación Científica y Técnica (Project Number CTQ2006-12552/BQU), and the Fundación SENECA (Project Number 08813/PI/08). C.M. Soto thanks the Ministerio de Educación y Ciencia for the grant received.

\section{REFERENCES}

[1] Bard, A.J.; Stratmann, M.; Fujihira, M.; Rusling, J.F. and Rubinstein, I.; Eds. Encyclopedia of Electrochemistry WilleyVCH: Weinheim, 2007.

[2] Bard, A.J.; Faulkner, L.R. Electrochemical Methods, Fundamental and Applications, 2nd ed.; Wiley: New York, 2001.

[3] Bartlett, P. N. Bioelectrochemistry, Fundamentals, Expermiental techniques and applications. Wiley: Chichester, 2008.

[4] Savéant, J.M. Molecular catalysis of electrochemical reactions. Mechanistic Aspects. Chem. Rev, 2008, 108, 2348-2378.

[5] Laviron, E.; in; Bard, A.J.; Ed. Electroanalytical Chemistry. Marcel Dekker: New York, 1982.

[6] Zanello, P. Inorganic Electrochemistry: theory, practise and application. Royal Society of Chemistry: Cambridge, 2003.

[7] Flinkea, H.O. in A.J. Bard, I. Rubinstein (Eds), Electroanalytical Chemisty; Marcel Dekker: New York, 1996.

[8] Chidsey, C.E.D. Free energy and temperature dependence of electron transfer at the metal-electrolyte Interface. Science, 1991, 251, 919-922.

[9] Rowe, G.K.; Creager, S.E., Redox and ion-pairing thermodynamics in self-assembled monolayers. Langmuir, 1991, 7, 2307-2312.

[10] Heering, H.A.; Mondal, M.S.; Amstrong, F.A. Using the pulsed nature of staircase cyclic voltammetry to determine interfacial electron-transfer rates of adsorbed species. Anal. Chem., 1999, 71, 174-182. 
[11] Komorsky-Lovric, S.; Lovric, M. Square-wave voltammetry of quasi-reversible surface redox reactions. J. Electroanal. Chem., 1995, 384, 115-122.

[12] Forster, R.J.; Faulkner, L.R. Electrochemistry of spontaneously adsorbed monolayers. Effects of solvent, potential, and temperature on electron transfer dynamics. Electrochemistry of spontaneously adsorbed monolayers. Effects of solvent, potential, and temperature on electron transfer dynamics. J. Am. Chem. Soc., 1994, 116, 54535461.

[13] González, J.; Abenza, N.; Molina, A. Analytical solutions of the multipotential pulse quasi-reversible Q-E-t and I-E-t responses of strongly adsorpted redox molecules. J. Electroanal. Chem., 2006, 596, 74-86.

[14] Abenza, N.; González, J.; Molina, A. General behaviour of the I-E and $\Delta \mathrm{I}-\mathrm{E}$ curves obtained when a multistep potential is applied to an electroactive monolayer. Electroanalysis, 2007, 19, 936-944.

[15] González, J.; Molina, A.; Abenza, N.; Serna, C.; Moreno, M. M. Square wave voltcoulometry: a new tool for the study of strongly adsorbed redox molecules. Anal. Chem., 2007, 79, 7580-7587.

[16] Mirceski, V.; Komorsky-Lovric, S.; Lovric, M. Square Wave Voltammetry, Theory and Application; Springer: Berlin, 2007.

[17] Jeuken, L.J.C.; McEvoy, J.P.; Armstrong, F.A., Insights into gated electron-transfer kinetics at the electrode-protein interface: A square wave voltammetry study of the blue copper protein azurin. J. Phys. Chem., 2002, 106, 2304-2313.

[18] Komorsky-Lovric, S.; Lovric, M. A peak current - scan rate relationship in staircase voltammetry of a surface redox reaction. Electroanalysis ,1996, 8, 959-962.

[19] González, J.; Soto, C.M.; Molina, A. Analytical I-E responses for several multistep potential techniques applied to an electrocatalytic process at mediator modified electrodes. Electrochim. Acta, 2009, doi:10.1016/j.electacta. 2009.05.068.

[20] Molina, A.; González, J. Cyclic reciprocal derivative chronopotentometry with power time currents applied to electrodes coated with electroactive molecular films. Influence of the reversibility. Langmuir, 2003, 19, 406-415.

[21] González, J.; Molina, A. cyclic reciprocal derivative chronopotentiometry with exponential time currents in the study of slow charge transfer processes between electrodes and redox adsorbates. Langmuir, 2001, 17, 5520-5526.

[22] González, J.; Molina, A. Charge-potential and capacitancepotential curves corresponding to reversible redox monolayers. $J$. Electroanal. Chem., 2003, 557, 157-165.

[23] González, J.; Molina, A. Linear sweep voltammetric and chronopotentiometric charge/potential curves for non reversible redox monolayers. J. Electroanal. Chem., 2005, 583, 184-192.

[24] Moreno, M.M.; Molina, A. Further applications of cyclic voltammetry in spherical electrodes. Coll. Czech. Chem. Commun., 2005, 70, 133-153.

[25] Molina, A.; Moreno, M.M.; Serna, C.; Lopez-Tenés, M.; González, J.; Abenza, N. Study of multicenter redox molecules with Square Wave Voltammetry. J. Phys. Chem., 2007, 111, 12446-12453.

[26] Osteryoung, J.; O'Dea, J.J.; in; Bard, A.J.; Ed. Square-wave voltammetry. Electroanalytical chemistry; Marcel Dekker: New York, 1986.

[27] Mirceski, V.; Gulaboski, R. The surface catalytic mechanism: a comparative study with square-wave and staircase cyclic voltammetry. J. Solid State Electrochem., 2003, 7, 157-165.

[28] Molina, A.; González, J. Particular time independent behaviour of the charge/potential and capacitance/potential responses of a quasireversible redox monolayer with chronopotentiometry with an exponential current. J. Electroanal. Chem., 2005, 585, 132-141.

[29] Limoges, B.; Savéant, J. M. Catalysis by immobilized redox enzymes. Diagnosis of inactivation and reactivation effects through odd cyclic voltammetric responses. J. Electroanal. Chem., 2004, $562,43-52$.

(c) González et al.; Licensee Bentham Open.

This is an open access article licensed under the terms of the Creative Commons Attribution Non-Commercial License (http: //creativecommons.org/licenses/by$\mathrm{nc} / 3.0 /$ ) which permits unrestricted, non-commercial use, distribution and reproduction in any medium, provided the work is properly cited. 